\title{
Multiple Complications Due to Subacute Suppurative Otitis Media
}

\author{
Brikena Qirjazi ${ }^{1}$ Dolores Bardhyli², Xhevair Hoxhallari² \\ 1 Department of ENT, Faculty of Medicine, University of Tirana, Tirana, Albania \\ 2Department of ENT, Tirana University Hospital Center, Tirana, Albania
}

\begin{abstract}
Subacute otitis media is a well-known pathology of ENT practice which is easily diagnosed and subsequently treated in the outpatient clinic. The rate of complications in acute otitis media is lower than in chronic otitis media. We present here the history of a young patient with subacute otitis media who developed both localized labyrinthitis and facial palsy requiring surgical treatment. We conclude that the treatment of subacute otitis media should be carefully monitored because complications may occur. Surgical treatment and pressure release can be efficient for the treatment of a peripheral facial palsy in the course of acute or subacute otitis media.
\end{abstract}

Key Words: Subacute otitis media, facial palsy, labyrinthitis

Received: 23.11.2011 Accepted: 13.02.2012

\section{Introduction}

The pathophysiology of the complications of the suppurative otitis media is still a matter of debate in the medical literature. The broad use of antibiotics has evidently decreased the incidence of complications, but independent of this, the morbidity and the mortality rate of these complications is still high (1). Thus, the decreasing incidence rate may lead to late diagnoses and therefore late treatment, which in turn may result in persistent sequelae.

Both purulent and serous labyrinthitis are complications of subacute purulent otitis media. The latter is thought to be a result of toxins and inflammatory mediators penetrating into the labyrinth. If not properly treated, it may lead to the purulent labyrinthitis. Meanwhile, facial palsy is a more frequent complication, especially in the adult population. There are several hypotheses that try to explain the mechanism of the facial palsy following a suppurative otitis media (2-4).

All these complications should be treated aggressively, because there is a risk for permanent damage. This paper presents the case of a young female patient who developed facial palsy and serous labyrinthitis after subacute otitis media.

\section{Case Report}

A 34-year-old female patient presented at a district hospital with a one-month history of tinnitus and mild pain in the right ear. The patient was diagnosed with acute otitis media and treated ambulatorily with antibiotics and nasal decongestants. According to the patient, the tinnitus did not stop and the pain increased. After three to four weeks of treatment, the patient started to complain of dizziness and vomiting. The pain was not present any longer, while the tinnitus was continuously disturbing. The patient was hospitalized and mannitol IV was added to the therapy. In Albania, mannitol is traditionally used as an osmotic diuretic to reduce fluid distention of the labyrinth. The neurologic examination showed no central involvement and added nothing to the overall picture of the disease.

At this point, the patient was sent to the University Hospital Center of Tirana, where she presented with pain in the right ear, spontaneous first grade nystagmus on the right and hearing loss on the right side. The audiogram on the day of presentation showed mixed hearing loss in the right ear, with an average bone conduction of 30-35 dB HL and an air-to-bone gap of around 40 $\mathrm{dB}$. An otoscopic evaluation showed swelling of the posteriorsuperior quadrant of the tympanic membrane. On the second day of treatment in the hospital in Tirana, a myringotomy was performed, where the release of pus under pressure was noted. The general condition of the patient improved. No antibiogram was requested; the subsequent therapy consisted of IV cefuroxime axetil, mannitol IV and a low dose of corticosteroids $(25 \mathrm{mg} /$ day). Two weeks later, swelling of the tympanic membrane was again noted and a grade IV House-Brackmann facial palsy was established. Under these circumstances, it was decided to perform an antrotomy. Granulation tissue in the antrum and tympanum was found during surgery and was cleaned out. The dose of corticosteroids was increased (up to $60 \mathrm{mg} /$ day with a tapered dose over three weeks) and gradually the facial palsy started to resolve. Three weeks after the surgical intervention, the facial palsy was evaluated as grade II (Figure 1). Pure tone audiometry showed mixed hearing loss in the right ear (Figure 2).

\section{Discussion}

The complications of the acute suppurative otitis media (ASOM) are defined as the spread of the infection behind the 


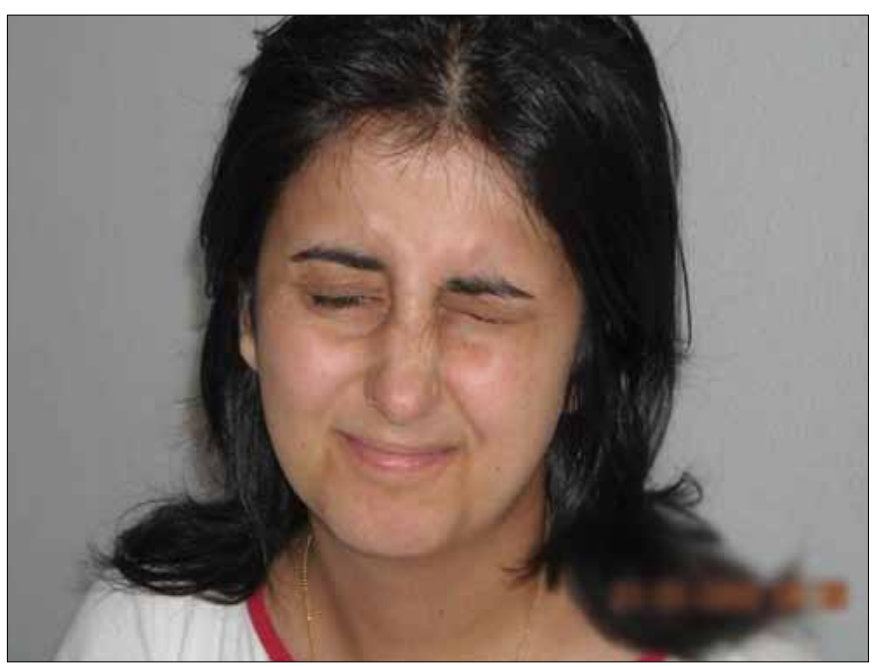

Figure 1. Grade II facial palsy, three weeks after surgery

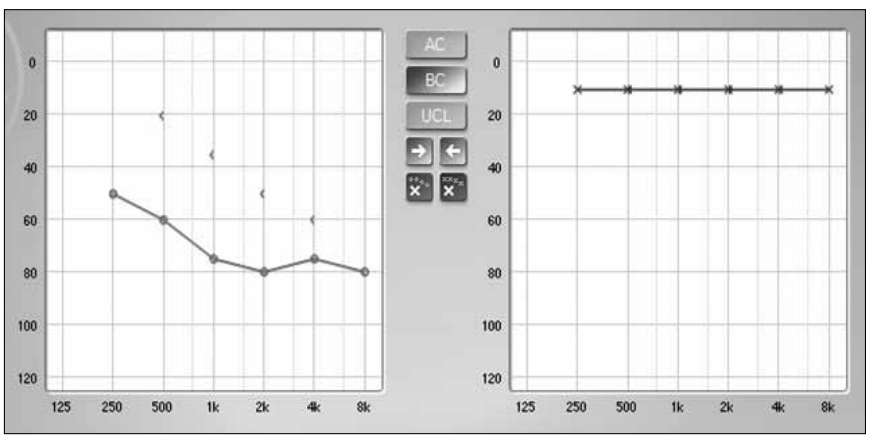

Figure 2. Pure tone audiometry on the final day of hospitalization

pneumatised area of the mastoid and the respective mucosa. The present case appears to be an interesting one because the multiple complications followed the course of a subacute suppurative otitis media (SSOM) rather than an ASOM. As the clinical presentation and the treatment choices for the complications are the same, we will below refer to them as a single entity. Both labyrinthitis and facial palsy are listed under the intratemporal complications of ASOM. Their treatment requires the use of IV antibiotics, and if the tympanic membrane is not perforated, they may also require a myringotomy.

The suggestive signs for possible involvement of the labyrinth are nystagmus, dizziness and sensori-neural hearing loss. These signs were all present in our case. A contrast MRI may be requested in order to obtain imaging of the inner ear; in our case, it was not needed as the clinical picture was complete and indicative. The treatment of the patient consisted of a combination of antibiotics and myringotomy; the latter confirmed the bacterial nature of the infection in the middle ear. Ludman (5) pointed out that the differentiation between the serous and the suppurative form of labyrinthitis is done retrospectively, depending on the residual vestibular and auditory function of the labyrinth. Without doubt, the clinical picture as well as the fluctuations in hearing loss are less prominent with the serous form that what is seen during purulent labyrinthitis. If the use of myringotomy and antibiotics does not give the expected results, corticosteroids are the treatment of choice; they are thought to provide some improvement to the residual auditory threshold (6). Unfortunately, the use of corticosteroids did not produce such results in our case. The patient was left with unilateral moderate to severe permanent hearing loss.

Facial palsy may occur through different mechanisms (7). It may be an inflammatory reaction to infection inside the Fallopian canal (4), but in other cases, the lack of a bony shell leads to direct pressure upon the nerve itself (2). The reactivation of a latent viral infection of the geniculate ganglion is another possible mechanism (8). For all these reasons, corticosteroids are highly recommended as a treatment modality for facial palsy; the results of this treatment are generally encouraging. This therapy was also used in our patient and the results of the combined therapy could be considered successful as they brought about a visible improvement of the peripheral facial palsy. Antrotomy was performed to secure better drainage of the middle ear, which would reduce the pressure upon the facial nerve.

The factors which predispose the development of complications in ASOM and the mechanisms responsible are still under debate in the medical literature. The presence of an infection for more than two weeks, the reactivation of an infection after two weeks, as well as the establishment of specific signs as dizziness or facial palsy (which direct toward a specific complication) may be considered as symptoms of alarm. The patients that develop complications are generally young, and this together with the risk of permanent damage should be taken into consideration during the treatment and follow-up of cases.

In this context, the proper use of antibiotics and close follow-up is important to prevent the spread of infection. Broad spectrum antibiotics are generally recommended in cases of suppurative otitis media. However, the use of antibiotics may not always protect against complications, or worse, it may mask the signs of complications (9). Under some circumstances, this might be explained by increased antibiotic resistance (10).

\section{Conflict of Interest}

No conflict of interest was declared by the authors.

\section{References}

1. Wetmore RF. Complications of otitis media. Pediatr Ann 2000;29:637-46.

2. Tschiassny K. Is facial palsy, when complicating cases of acute otitis media, indicative for immediate mastoid operation? Cincinnati Med 1944;25:262-6.

3. Miehlke A. Surgical classification of the paralysis of the facial nerve, with guidelines for differential diagnosis and therapy. In: Miehlke A, ed. Surgery of the Facial Nerve. Munich: Urban \&Schwarzenberg 197;52-8.

4. Antoli-Candela E, Stewart TJ. The pathophysiology of otologic facial paralysis. Otolaryngol Clin North Am 1974;7:309-30.

5. Ludman H. Complications of suppurative otitis media. In: Kerr AG, ed. Scott-Brown's Otolaryngology, 5th edition, London: Butterworths 1987;264-91.

6. Kappaport JM, Bnatt SM, Burkard KF, Merchant SN, Nadol JB Jr. Prevention of hearing loss in experimental meningitis by administration of dexamethasone and ketorolac. J Infect Dis 1999; 179:264-8. [CrossRef]

7. Luca O, Radaelli de Zinis, Paolo Gamba, Christiano Balzanelli. Acute Otitis media ad facial nerve paralyses in adults. Otology and neurotology 2003;24:113-7.

8. Adour KK, Hetzler DG. Current medical treatment of facial palsy. Am J Otol 1984;5:499-502.

9. Lin YS, Lin LC, Lee FP, Lee KJ. The prevalence of chronic otitis media and its complications rates in teenagers and adults. Otolaryngol Head \& Neck Surg 2009;140:165-70. [CrossRef]

10. Vergison A. Microbiology of otitis media: A moving target. Vaccine 2008;26:G5-10. [CrossRef] 